\title{
Application of Rough Set, GSM and MSM to Analyze Learning Outcome-An Example of Introduction to Education
}

\author{
Hui-Chung Ho1, Woody Jann-Der Fann², Hsiu-Jye Chiang3, Phung-Tuyen Nguyen4, \\ Duc-Hieu Pham ${ }^{5}$, Phuoc-Hai Nguyen ${ }^{4}$, Masatake Nagai6 \\ ${ }^{1}$ Retired, National Taichung University of Education, Taiwan \\ ${ }^{2}$ Ling-Tung University, Taiwan \\ ${ }^{3}$ National United University, Taiwan \\ ${ }^{4}$ Kien Giang Teacher Training College, Rach Gia, Vietnam \\ ${ }^{5}$ Hanoi Pedagogical University 2, Xuan Hoa, Vietnam \\ ${ }^{6}$ Retired, Teikyo University, Tokyo, Japan \\ Email: A22263181@yahoo.com.tw
}

Received 25 August 2015; accepted 16 February 2016; published 19 February 2016

Copyright (C) 2016 by authors and Scientific Research Publishing Inc.

This work is licensed under the Creative Commons Attribution International License (CC BY).

http://creativecommons.org/licenses/by/4.0/

c) (7) Open Access

\begin{abstract}
Introduction to education is one of the basic courses in teacher education professional education, it covers a wide range of subjects. Thus, in order to practice the management teaching goals, the interdisciplinary developed mathematical tools are applied for the study. The participants of this study are students in course of introduction to education, and the research instruments applied are rough set, grey structural modeling (GSM), and matrix based-structural modeling (MSM). The purposes of this paper are: 1) To logically analyze educational datasets to practice the scientific traits in education; 2) To benefit from directed hierarchical analysis to identify and propose action planning; 3) To construct core-oriented educational structure as the criterion-reference for onelesson-multiple-design and to provide the whole scope and visualized analysis with GSM and MSM.
\end{abstract}

\section{Keywords}

Introduction to Education, Rough Set, GSM, MSM, One-Lesson-Multiple-Design

\section{Introduction}

"If a person neglects education, he will miss the future”. In the 21st century, education carries more the profes-

How to cite this paper: Ho, H.-C., Fann, W.J.-D., Chiang, H.-J., Nguyen, P.-T., Pham, D.-H., Nguyen, P.-H. and Nagai, M. (2016) Application of Rough Set, GSM and MSM to Analyze Learning Outcome-An Example of Introduction to Education. Journal of Intelligent Learning Systems and Applications, 8, 23-38. http://dx.doi.org/10.4236/jilsa.2016.81003 
sionalism and modernity than ever. The course of introduction to education is one of the professional basic education courses, covering a wide range, such as philosophy, psychology, sociology, and shifting territory when accompanied by new elements such as digital migration technology, ecology and cultural anthropology. For students majoring in education of regular training 4 years and those students studying education, they must have been aware of the overarching and comprehensive professional education after one semester, it is clear not simple. Simultaneously, with those responsible for teaching this course, challenges to achieve effective as anticipated clearly not small in the context of learners bring different traits include: motive for registration, belonging to different departments, as college students and graduate students, study attitude are also different [1].

To understand and manage the effectiveness of teaching and learning in course of introduction to education, this study has applied the mathematical tools of research and development towards transdisciplinary research on subjects for students to choose school this subject, aim to: 1) Understand the structural concept of the course focused on the topic of the course and the course, 2) Discriminate the structural conceptual attribute and the development, open this broad concepts, 3) Provide a basis for teaching standards with different textures for the same subject [2]. Research tools were developed based on mathematical principles, such as Rough set, GSM, MSM, and applied in the field of education [3] [4], the results obtained: 1) The value of education practice reflects educational operating with science; 2) Educational thinking is transformed into mathematical logic, structural characterization analysis have to be stratification; 3) Provide exchange channel related educational diagnosis and meta-discourse.

Due to the social requirements of network and postmodernism multi-awareness, effectiveness of education and educational standardization has become the key point interested in the renewal and reform of education. Applying mathematical models to combine the development of science and technology in educational research activities conducive to the development, conversion and modernization as well as receiving the evaluation and supervision from community on education. Therefore, the purpose of the study is to: 1) Recognize the right way of distinctness of the original act in the learning process, build knowledge structures according to the trend of learner as nuclear; 2) This study is scientific and so that possible replicative. The progress of research can be mathematized and visualized from examining the data and calculations, mathematical reasoning to structural analysis; 3) Propose a new model of educational research, that is the educational research project with the implementation process combining qualitative and quantitative analyses.

\section{Mathematical Tools}

Based on the curriculum of domestic education research methodology, the educational academic and practical researches, educational researches are divided into qualitative and quantitative research paradigms, the former as case study, ethnographic, field study, and narrative analysis, the latter covers the design and construction survey questionnaire, data verification, statistical analysis and prediction. This is to enhance the communication of educational research, the trustworthiness of findings, as well as in response to interdisciplinary trend, the use of mathematically based system of symbolic logic are used as the characterization and analysis tools.

Based on the context of the development of education having coherence and educational research with transferability, using mathematical characterization and analysis tools, in order to: 1) Develop result analysis and interpretation of the data trend; 2) Morphological structural visualization; 3) Propose the design of new researches which have systematic, experimentation, and interpretation. Therefore, the following is introduction of mathematical tools used. This study applied Rough Set Theory of Pawlak [5] and Grey System Theory of Deng [6] as the mathematical models of basic development, intended to mathematize scientific form analysis and characterize the practical problem researches of education.

\subsection{Rough Set Theory}

The rough set theory was introduced by Pawlak in the early 1980s as a mathematical tool to deal with uncertainty. It does not need to be given quantity description or statistical probability distribution of some characteristics or attributes in advance and does not have to obey any assumptions. Rough set theory only says that the set of objects analyzed imply the knowledge and knowledge is considered a classification ability of objects [7]. "Reduct" and "core" are important concepts in the rough set theory. Reduct is those minimal attribute sets of information system, which keep the same classification capability with original attribute set. The aim of feature subset selection is to find out a minimum set of relevant attributes that describe the dataset as well as all original attributes do. The basic concepts of rough set are introduced in the following section [8] [9]. 


\subsubsection{Information System}

Formally, it is an information system, and can be seen as a system:

$$
I S=(U, R, V, f)
$$

where: i. $U=\left\{x_{1}, x_{2}, \cdots, x_{m}\right\}$ is the universe, a non-empty set of finite objects.

ii. $R=\left\{r_{1}, r_{2}, \cdots, r_{n}\right\}$ is a non-empty, finite set of attributes.

iii. $V=\left\{V_{1}, V_{2}, \cdots, V_{n}\right\}, V_{i}$ is the range of attribute $r_{i}$.

iv. $f$ is a set of $f_{r}$, information function.

Each attribute $r_{i} \in R$ defines an information function:

$$
f_{r_{i}}: U \rightarrow V_{i}
$$

\subsubsection{Indiscernibility Relation}

According to rough set theory, for every set of attributes $B \subset A$, an indiscernibility relation $\operatorname{Ind}(B)$ is defined: two objects $x_{i}$ and $x_{j}$ are indiscernible by the set of attributes $B$ in $A$, if $b\left(x_{i}\right)=b\left(x_{j}\right)$ for every $b \subset B$. The equivalence class of $\operatorname{Ind}(B)$ is called elementary set in $B$ because it represents the smallest discernible groups of objects. For any element $x_{i}$ of $U$, the equivalence class of $x_{i}$ in relation $\operatorname{Ind}(B)$ is represented as $\left[x_{i}\right]_{\operatorname{Ind}(B)}$.

\subsubsection{Upper and Lower Approximations}

The rough set approach to data analysis hinges on two basic concepts, namely the lower and the upper approximations of a set, lower approximation refers to the elements that surely belong to the set, and upper approximation refers to the elements that possibly belong to the set.

Let $X \subset U$, the lower approximation of $X$ in $B$ denoted as $\underline{B X}(B \subseteq A)$,

$$
\underline{B X}=\left\{x_{i} \in U \mid\left[X_{i}\right]_{\operatorname{Ind}(B)} \subset X\right\}
$$

the upper approximation of the set $X$ is:

$$
\overline{B X}=\left\{x_{i} \in U \mid\left[x_{i}\right]_{\operatorname{Ind}(B)} \cap X \neq \phi\right\}
$$

Some other concepts:

$$
\begin{aligned}
& B N X=\overline{B X}-\underline{B X} \text { is called a boundary of } X ; \\
& \operatorname{POS}_{B}(X)=\underline{B X} \text {, called the } B \text {-positive region; } \\
& N E G_{B}(X)=U-\underline{B X} \text {, called the } B \text {-negative region. }
\end{aligned}
$$

\subsubsection{Dependence and Significance of Attribute}

Let $C$ and $D$ be two indiscernibility relations in set $S, C$ is condition attribute and $D$ is decision attribute.

$$
\operatorname{pos}_{C}(D)=\bigcup_{x \in X / \operatorname{Ind}(D)} \underline{C X}
$$

The dependence $\gamma_{C}(D)$ of decision attribute $D$ on condition attribute $C$ is defined as:

$$
\gamma_{C}(D)=\frac{\operatorname{pos}_{C}(D)}{|U|}
$$

The dependence of attribute decides significance of attribute. The significance of attribute $r_{i} \in C$ is defined:

$$
\sigma_{(C, D)}\left(r_{i}\right)=\frac{\gamma_{C}(D)-\gamma_{C-\left\{r_{i}\right\}}(D)}{\gamma_{C}(D)}=1-\frac{\gamma_{C-\left\{r_{i}\right\}}(D)}{\gamma_{C}(D)}
$$




\subsubsection{Independence of Attributes}

If $\operatorname{Ind}(R)=\operatorname{Ind}\left(R-r_{i}\right)$, then the attribute $r_{i}$ is called superfluous. Otherwise, the attribute $r_{i}$ is in dispensable in $R$. Set $B$ is independent if all its attributes are indispensable.

\subsubsection{Core and Reduct of Attributes}

If the set of attributes is dependent, it can be interested in finding all possible minimal subsets of attributes, which lead to the same number of elementary sets as the whole set of attributes (called reducts) and in finding the set of all indispensable attributes (called core). The reduct is the essential part of an IS, which can discern all objects discernible by the original IS. The core is the common part of all reducts.

$$
\text { Core }(I S)=\bigcap \operatorname{red}(\text { IS })
$$

where red(IS) is the reduct of information system IS.

\subsection{Grey Structural Modeling (GSM)}

GSM was also proposed by Nagai in 2005, which is based on grey system theory and like interpretive structural modeling, its purpose is to analyze structure of system because it can find out the relationship from observed value indirectly such as statistical graphical modeling [10] [11]. GSM was proposed based on proposing grey relational analysis (GRA) to calculate localized grey relational grade, globalized grey relational grade and grey relational ordinal. Then based on grey relational grade to compare with each other, when one of the parties had a larger value, it was identified as more important item, and became criteria of structural system arrangement.

In grey system theory, GRA is an effective mathematical tool to treat the uncertain, multiple, discrete and incomplete information. This study refers the localized grey relational grade which is proposed by Nagai [12].

The original vectors in GRA:

The reference vector $x_{0}$ and inspected vectors of original data $x_{i}$ are established as followed:

$$
\begin{aligned}
x_{0} & =\left(x_{0}(1), x_{0}(2), \cdots, x_{0}(n)\right) \\
x_{1} & =\left(x_{1}(1), x_{1}(2), \cdots, x_{1}(n)\right) \\
x_{2} & =\left(x_{2}(1), x_{2}(2), \cdots, x_{2}(n)\right) \\
& \vdots \\
x_{m} & =\left(x_{m}(1), x_{m}(2), \cdots, x_{m}(n)\right), \text { where } i=1,2, \cdots, m ; m \in N
\end{aligned}
$$

The formula of localized grey relational grade is:

$$
\gamma_{o i}=\frac{\max _{\forall i}\left\|x_{0}-x_{i}\right\|_{\rho}-\left\|x_{0}-x_{i}\right\|_{\rho}}{\max _{\forall i}\left\|x_{0}-x_{i}\right\|_{\rho}-\min _{\forall i}\left\|x_{0}-x_{i}\right\|_{\rho}}
$$

And formula of globalized grey relational grade is:

$$
\gamma_{i j}=1-\frac{\left\|x_{i}-x_{j}\right\|_{\rho}}{\max _{\forall i} \max _{\forall j}\left\|x_{i}-x_{j}\right\|_{\rho}}
$$

GSM draws digraph by using three parameters: distinguish coefficient $\rho$ which decides the basic composition of digraph, class coefficient $\theta$ which gives the hierarchy, and path coefficient $\psi$ which gives an ordered pair of element arrows. In this paper, $\rho=2$ is used that means Euclidean distance is applied.

\subsection{Matrix Based Structure Modeling (MSM)}

MSM was proposed by Nagai in 2013 [13] [14], it was intended to generate an overall association between matrices and reconfigure multiple matrices into square design, in order to construct a new relationship between the respective sets of generation, and visualize the entirety of matrix and the logical relationship of local clusters.

Based on the multi-matrix method of interpretative structural modeling, it is simply known as multi-matrix 
interpretative structural model.

The combined set of system:

The combined set

$$
M=\bigcup M_{i}
$$

where $M_{i}$ is the concept set matrix, it can be all set of material objects, it can also be the set of all attributes such as set of students, set of questions, set of learning concept, set of goods, set of merchandise category, set of commodity components, set of commodity production conditions, etc.

The structure system theory of MSM is contracted as the following:

Establish the structure system theory of MSM

$$
W=M S M(M, T, f)
$$

where i. $M=\bigcup M_{i}$ is a combined matrix of factor set,

ii. $T=\bigcup_{r \in M} T_{r}$ is structure matrix,

iii. $f: M \times M \rightarrow T$ is reachability function,

$$
\begin{gathered}
M \times M=\left\{\left(m_{i}, m_{j}\right) \mid m_{i}, m_{j} \in M\right\} \\
f\left(m_{i}, m_{j}\right)=m_{i j} \in T_{r} .
\end{gathered}
$$

Because $f$ is a reachability function, so $f$ satisfies the following:

1) Reflexive law: $f\left(m_{i}, m_{i}\right)=1 \Leftrightarrow m_{i} \rightarrow m_{i}, \forall i$

2) Anti-symmetric law:

$$
\begin{aligned}
& f\left(m_{i}, m_{j}\right)=f\left(m_{j}, m_{i}\right)=1 \\
& \Leftrightarrow m_{i} \rightarrow m_{j}, m_{j} \rightarrow m_{i} \Rightarrow m_{i} \leftrightarrow m_{j}, \forall i \neq j \\
& \Leftrightarrow m_{i}=m_{j}
\end{aligned}
$$

3) Transitive law:

$$
\begin{aligned}
& f\left(m_{i}, m_{j}\right)=1, f\left(m_{j}, m_{k}\right)=1 \\
& \Rightarrow f\left(m_{i}, m_{k}\right)=1 \quad, \forall i \neq j \neq k \\
& \Leftrightarrow m_{i} \rightarrow m_{j}, m_{j} \rightarrow m_{k} \\
& \Rightarrow m_{i} \rightarrow m_{k}
\end{aligned}
$$

In practice, the MSM cluster matrix calculates amount and scale size using structural new relation matrix as purpose based on the generating of multiple matrix relation and the creating of organization structure, the first one is derived from the relationship between the various elements of attributes, and second one, it is the cluster related structure of the purpose orientation [15] [16].

\section{Analysis of Practical Example}

The research objects are 21 third-year students of higher education and 15 syllabus researchers, the former has been the introduction to education of the first year, the latter is chapters in detail of the introduction to education of this semester, aimed to: 1) Understand two learning objects to explain the differences, 2) Form the structure of unit nuclear knowledge, 3) Make a standard reference basis for the teaching of introduction to education in future.

\subsection{Research Process}

The research process is divided into qualitative and quantitative analysis stages (see Figure 1). Firstly, the students select the important concepts related to basic theory of introduction to education; secondly, using the number values of 1 to 9 to fill out the questionnaire and analysis, the greater the value represents the more con- 


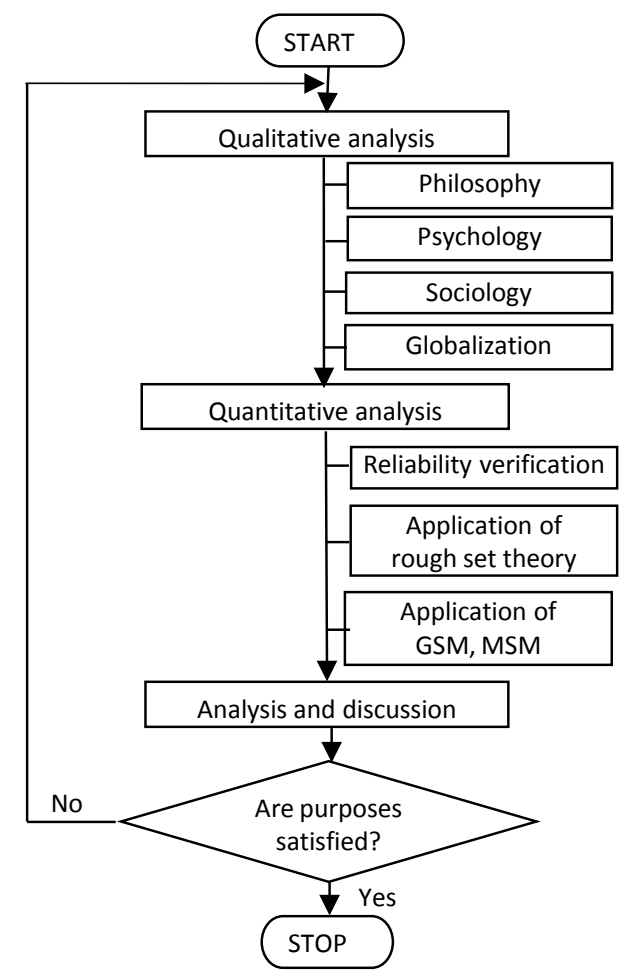

Figure 1. Research process.

cept learning difficulty, on the contrary, the concept learning easier. Thirdly, after testing the reliability of data, using rough set to excavate information; the GSM constitutes isomorphism of "core", analyzes body of knowledge and structure; MSM characterizes its structural relation. Finally, according to the proposal of structural analysis and directed hierarchy diagram, the diagram is explained.

\subsection{Q-Matrix}

Towards globalization, technology, and Internet of Things (IOT) era, introduction to the basic theory of education from the traditional philosophy, psychology, sociology, new cultural anthropology, ecology, science and technology and culture, etc., are only selecting the concepts on the first four fields as follows (see Table 1): 1) Philosophy-epistemology, metaphysics and theory of value; 2) Sociology, cultural anthropology—structural functionalism, system theory, educational political science, educational finance and local/regional culture; 3) Psychology—cognitive science, personality development, positive psychology.

It is worth mentioning: Educational political science and educational finance of sociology aim to: 1) Initiated by the post-modern pluralism, the overall educational practice strategizing becomes necessary, and thus more and more important in educational political science; 2) Facing the globalization of competing interests, profit trends, while input and output performance become the focus of education, on the other hand under the austerity policies being squeezed available funding for education, educational return on investment become truth to be faced; 3) The World is Flat [17], "investing in education, investing in the future," human capital, human development and quality of financial education is closely related.

Next, combining concepts and pedagogy to constitute the Q matrix (Table 1), both relations generate principles: 1) Use the binary to represent the relationship between the elements, 2) Distinguish the meanings of concept, expansion of knowledge with its knowledge system and the difficulty of the justification, 3) Teaching model assigns teacher to guide meaningful teaching methods, design the situations of teacher and learners engaged in discovery teaching method, goal setting, problem solving compromise-oriented legislation.

Finally, combining graph theory and interpretive structural modeling (ISM) [18] to build a mapping analysis and visualize the hierarchical structure (see Figure 2), conduct the useful classroom, the text with multiple designs, diagnosis and establish the basis for later reflection. 


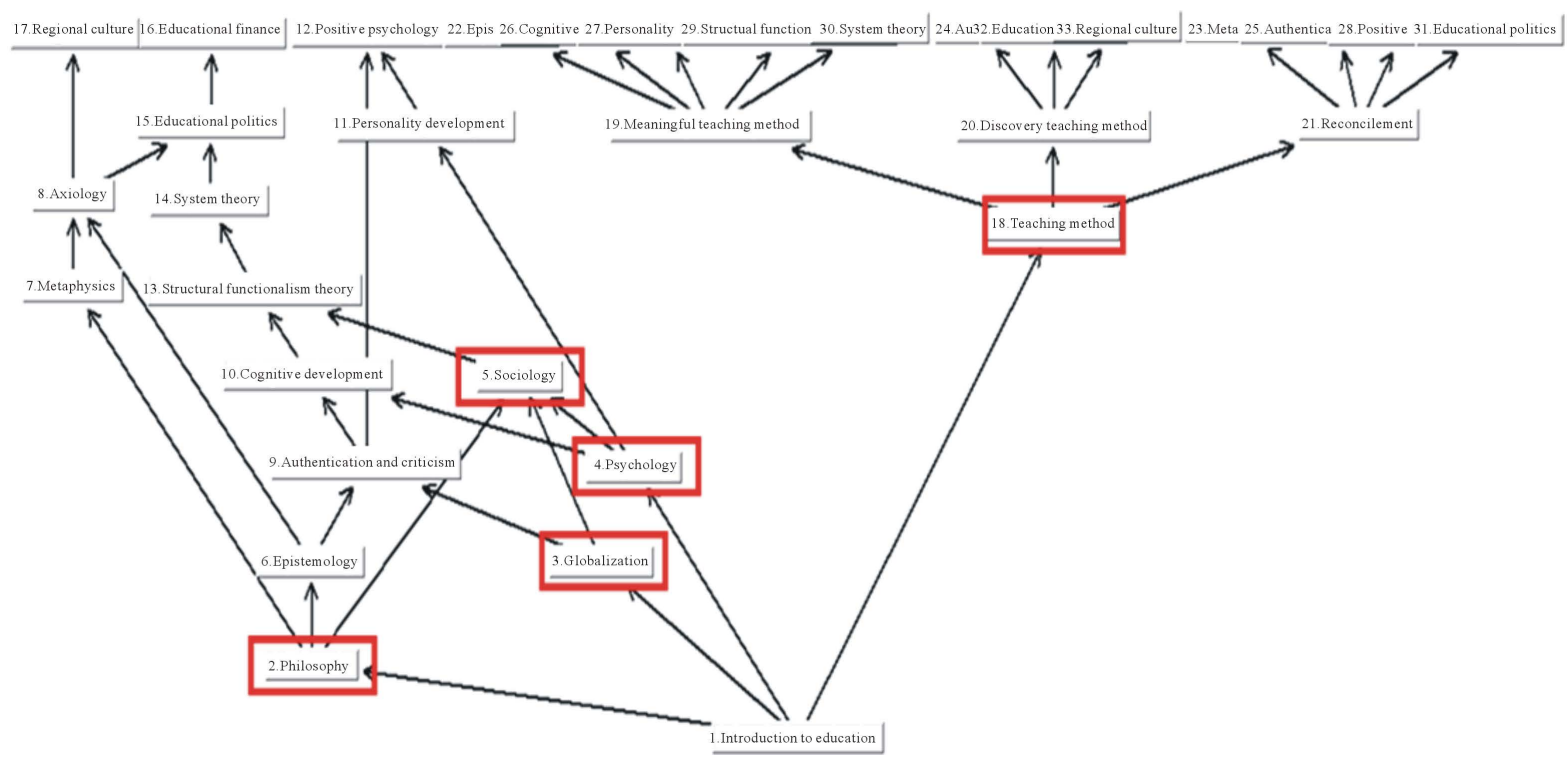

Figure 2. ISM diagram of concepts-teaching method.

\section{Table 1. Q-matrix.}

\begin{tabular}{|c|c|c|c|c|}
\hline Subject & Concept & Meaning teaching & Discovery teaching & Eclectic teaching \\
\hline \multirow{3}{*}{ Philosophy } & Epistemology & 1 & 0 & 0 \\
\hline & Metaphysics & 0 & 0 & 1 \\
\hline & Axiology & 0 & 1 & 0 \\
\hline \multirow{3}{*}{ Psychology } & Cognitive development & 1 & 0 & 0 \\
\hline & Personality development & 1 & 0 & 0 \\
\hline & Positive psychology & 0 & 0 & 1 \\
\hline \multirow{4}{*}{ Sociology } & Structural functionalism & 1 & 0 & 0 \\
\hline & System theory & 1 & 0 & 0 \\
\hline & Educational politics & 0 & 0 & 1 \\
\hline & Educational finance & 0 & 1 & 0 \\
\hline \multirow{2}{*}{ Globalization } & Regional culture & 0 & 1 & 0 \\
\hline & Authenticn and criticism & 0 & 0 & 1 \\
\hline
\end{tabular}

\subsection{Data Processing}

Firstly, the data of the four-year undergraduate education including 21 third-year students and 15 syllabus researchers in primary education course, all are tested for reliability, Cronbach's alpha coefficients are shown in Table 2, all of them are available for use (clean data). Bryman \& Cramer (1997) [19] believed that if Cronbach's alpha coefficient is greater than 0.80 it indicates a set of high reliability data.

Secondly, based on grey relational analysis the degree of relation between the discrete elements which is calculated by formula of Nagai [20], the orders of element are determined (see the Tables 3-6). Using ROSE 2 software, from a common intersection of sets of reducts, the "Core" is found out to construct orientation of structural analysis, and identify it as an available teaching reference standard. Moreover, submitting an alternative educational dialogue.

Finally, based on "We Are Student-Centered” (WASC) [21] and implemented performance accomplishments 
Table 2. Cronbach's alpha coefficients of sets of data.

\begin{tabular}{ccc}
\hline Objectltable type & S-P table & P-S table \\
\hline Third year student & 0.82 & 0.86 \\
Researcher & 0.82 & 0.92 \\
\hline
\end{tabular}

Table 3. Localized grey relational grade of educational students/undergraduates.

\begin{tabular}{|c|c|c|c|c|c|c|c|c|c|c|c|c|c|}
\hline Student $\backslash$ Concept & A9 & A13 & A10 & A14 & A8 & A6 & A3 & A7 & A11 & A12 & A5 & A4 & Camm \\
\hline Max & 9 & 9 & 9 & 9 & 9 & 9 & 9 & 9 & 9 & 9 & 9 & 7 & जитाти \\
\hline S7 & 9 & 7 & 7 & 8 & 9 & 7 & 9 & 8 & 8 & 9 & 8 & 7 & 1.00 \\
\hline S2 & 8 & 8 & 8 & 9 & 8 & 8 & 8 & 6 & 7 & 7 & 7 & 7 & 0.93 \\
\hline S4 & 9 & 9 & 6 & 5 & 8 & 9 & 5 & 7 & 5 & 6 & 7 & 4 & 0.69 \\
\hline S15 & 9 & 5 & 8 & 3 & 9 & 7 & 8 & 9 & 6 & 7 & 6 & 5 & 0.69 \\
\hline S8 & 8 & 4 & 9 & 4 & 9 & 9 & 8 & 4 & 6 & 7 & 8 & 5 & 0.66 \\
\hline S16 & 7 & 7 & 7 & 7 & 7 & 6 & 5 & 5 & 6 & 6 & 5 & 4 & 0.63 \\
\hline S18 & 7 & 3 & 6 & 5 & 9 & 7 & 8 & 8 & 8 & 5 & 6 & 4 & 0.62 \\
\hline S13 & 8 & 8 & 7 & 8 & 9 & 8 & 6 & 9 & 3 & 2 & 7 & 5 & 0.61 \\
\hline S21 & 8 & 6 & 8 & 7 & 3 & 9 & 3 & 7 & 9 & 7 & 4 & 6 & 0.58 \\
\hline S11 & 9 & 5 & 8 & 3 & 8 & 5 & 5 & 8 & 6 & 7 & 4 & 3 & 0.53 \\
\hline S10 & 9 & 6 & 7 & 5 & 8 & 5 & 5 & 9 & 3 & 2 & 6 & 5 & 0.48 \\
\hline S9 & 6 & 4 & 5 & 6 & 9 & 7 & 3 & 8 & 8 & 9 & 3 & 2 & 0.48 \\
\hline S17 & 7 & 5 & 6 & 5 & 8 & 9 & 4 & 9 & 4 & 3 & 7 & 1 & 0.45 \\
\hline S6 & 8 & 2 & 6 & 4 & 8 & 4 & 7 & 5 & 4 & 4 & 7 & 7 & 0.43 \\
\hline S5 & 8 & 4 & 9 & 4 & 7 & 3 & 7 & 6 & 2 & 3 & 9 & 3 & 0.38 \\
\hline S19 & 9 & 6 & 8 & 3 & 6 & 6 & 6 & 4 & 6 & 2 & 3 & 2 & 0.36 \\
\hline S12 & 7 & 2 & 5 & 3 & 6 & 6 & 5 & 3 & 7 & 7 & 4 & 1 & 0.30 \\
\hline S1 & 4 & 6 & 7 & 7 & 5 & 6 & 5 & 5 & 2 & 3 & 3 & 1 & 0.28 \\
\hline S3 & 6 & 6 & 7 & 7 & 3 & 4 & 2 & 3 & 6 & 4 & 2 & 2 & 0.24 \\
\hline S14 & 6 & 4 & 8 & 2 & 3 & 5 & 2 & 1 & 4 & 1 & 5 & 7 & 0.11 \\
\hline S20 & 5 & 3 & 2 & 4 & 5 & 1 & 5 & 6 & 2 & 1 & 2 & 1 & 0.00 \\
\hline
\end{tabular}

Table 4. Localized grey relational grade of concepts in educational student/four-year undergraduates.

\begin{tabular}{cccccccccccccccccccccccccc}
\hline Concept|Student & S1 & S2 & S3 & S4 & S5 & S6 & S7 & S8 & S9 & S10 & S11 & S12 & S13 & S14 & S15 & S16 & S17 & S18 & S19 & S20 & S21 & Gamma \\
Max & 7 & 9 & 7 & 9 & 9 & 8 & 9 & 9 & 9 & 9 & 9 & 7 & 9 & 8 & 9 & 7 & 9 & 9 & 9 & 6 & 9 & \\
\hline A9 & 4 & 8 & 6 & 9 & 8 & 8 & 9 & 8 & 6 & 9 & 9 & 7 & 8 & 6 & 9 & 7 & 7 & 7 & 9 & 5 & 8 & $\mathbf{1 . 0 0}$ \\
A13 & 6 & 8 & 6 & 9 & 4 & 2 & 7 & 4 & 4 & 6 & 5 & 2 & 8 & 4 & 5 & 7 & 5 & 3 & 6 & 3 & 6 & $\mathbf{0 . 9 2}$ \\
A10 & 7 & 8 & 7 & 6 & 9 & 6 & 7 & 9 & 5 & 7 & 8 & 5 & 7 & 8 & 8 & 7 & 6 & 6 & 8 & 2 & 8 & $\mathbf{0 . 9 1}$ \\
A14 & 7 & 9 & 7 & 5 & 4 & 4 & 8 & 4 & 6 & 5 & 3 & 3 & 8 & 2 & 3 & 7 & 5 & 5 & 3 & 4 & 7 & $\mathbf{0 . 7 9}$ \\
A8 & 5 & 8 & 3 & 8 & 7 & 8 & 9 & 9 & 9 & 8 & 8 & 6 & 9 & 3 & 9 & 7 & 8 & 9 & 6 & 5 & 3 & $\mathbf{0 . 7 1}$ \\
A6 & 6 & 8 & 4 & 9 & 3 & 4 & 7 & 9 & 7 & 5 & 5 & 6 & 8 & 5 & 7 & 6 & 9 & 7 & 6 & 1 & 9 & $\mathbf{0 . 5 0}$ \\
A3 & 5 & 8 & 2 & 5 & 7 & 7 & 9 & 8 & 3 & 5 & 5 & 5 & 6 & 2 & 8 & 5 & 4 & 8 & 6 & 5 & 3 & $\mathbf{0 . 4 9}$ \\
A7 & 5 & 6 & 3 & 7 & 6 & 5 & 8 & 4 & 8 & 9 & 8 & 3 & 9 & 1 & 9 & 5 & 9 & 8 & 4 & 6 & 7 & $\mathbf{0 . 4 5}$ \\
A11 & 2 & 7 & 6 & 5 & 2 & 4 & 8 & 6 & 8 & 3 & 6 & 7 & 3 & 4 & 6 & 6 & 4 & 8 & 6 & 2 & 9 & $\mathbf{0 . 4 3}$ \\
A12 & 3 & 7 & 4 & 6 & 3 & 4 & 9 & 7 & 9 & 2 & 7 & 7 & 2 & 1 & 7 & 6 & 3 & 5 & 2 & 1 & 7 & $\mathbf{0 . 4 1}$ \\
A5 & 3 & 7 & 2 & 7 & 9 & 7 & 8 & 8 & 3 & 6 & 4 & 4 & 7 & 5 & 6 & 5 & 7 & 6 & 3 & 2 & 4 & $\mathbf{0 . 2 7}$ \\
A4 & 1 & 7 & 2 & 4 & 3 & 7 & 7 & 5 & 2 & 5 & 3 & 1 & 5 & 7 & 5 & 4 & 1 & 4 & 2 & 1 & 6 & $\mathbf{0 . 0 0}$ \\
\hline
\end{tabular}


Table 5. Localized grey relational grade of master degree program researchers in education.

\begin{tabular}{cccccccccccccccc}
\hline StudentConcept & A3 & A4 & A5 & A13 & A6 & A7 & A8 & A9 & A10 & A11 & A12 & A14 & Gamma \\
Max & 9 & 9 & 9 & 9 & 9 & 9 & 9 & 9 & 9 & 6 & 9 & 7 & \\
S1 & 9 & 9 & 9 & 9 & 9 & 9 & 9 & 8 & 9 & 6 & 6 & 7 & $\mathbf{1 . 0 0}$ \\
S2 & 9 & 9 & 9 & 6 & 9 & 7 & 6 & 4 & 3 & 6 & 9 & 6 & $\mathbf{0 . 5 8}$ \\
S3 & 9 & 9 & 9 & 7 & 9 & 3 & 7 & 5 & 6 & 4 & 7 & 4 & $\mathbf{0 . 5 8}$ \\
S4 & 8 & 9 & 7 & 9 & 9 & 9 & 6 & 4 & 5 & 6 & 3 & 4 & $\mathbf{0 . 5 3}$ \\
S5 & 9 & 9 & 9 & 9 & 8 & 4 & 5 & 6 & 7 & 3 & 8 & 1 & $\mathbf{0 . 5 2}$ \\
S6 & 8 & 8 & 8 & 9 & 5 & 6 & 7 & 9 & 6 & 4 & 4 & 1 & $\mathbf{0 . 5 1}$ \\
S7 & 9 & 9 & 9 & 6 & 6 & 8 & 9 & 3 & 5 & 4 & 6 & 2 & $\mathbf{0 . 5 0}$ \\
S8 & 9 & 9 & 7 & 7 & 7 & 7 & 7 & 4 & 4 & 2 & 4 & 2 & $\mathbf{0 . 4 1}$ \\
S9 & 8 & 8 & 8 & 4 & 7 & 7 & 4 & 6 & 5 & 5 & 3 & 3 & $\mathbf{0 . 4 0}$ \\
S10 & 9 & 9 & 9 & 5 & 8 & 3 & 1 & 5 & 6 & 4 & 4 & 2 & $\mathbf{0 . 2 5}$ \\
S11 & 7 & 2 & 7 & 8 & 5 & 7 & 6 & 4 & 3 & 2 & 7 & 1 & $\mathbf{0 . 2 3}$ \\
S12 & 8 & 6 & 6 & 6 & 6 & 4 & 6 & 4 & 1 & 2 & 4 & 2 & $\mathbf{0 . 1 8}$ \\
S13 & 3 & 3 & 4 & 3 & 8 & 3 & 4 & 7 & 6 & 3 & 6 & 4 & $\mathbf{0 . 1 6}$ \\
S14 & 9 & 7 & 5 & 4 & 7 & 2 & 1 & 4 & 5 & 3 & 3 & 1 & $\mathbf{0 . 0 5}$ \\
S15 & 6 & 3 & 6 & 6 & 7 & 4 & 7 & 1 & 1 & 6 & 2 & 1 & $\mathbf{0 . 0 0}$ \\
\hline
\end{tabular}

Table 6. Localized grey relational grade of concepts in researchers in education/master degree program.

\begin{tabular}{cccccccccccccccccc}
\hline ConceptIStudent & S1 & S2 & S3 & S4 & S5 & S6 & S7 & S8 & S9 & S10 & S11 & S12 & S13 & S14 & S15 & Gamma \\
Max & 9 & 9 & 9 & 9 & 9 & 9 & 9 & 9 & 8 & 9 & 8 & 8 & 8 & 9 & 7 & \\
\hline A3 & 9 & 9 & 9 & 8 & 9 & 8 & 9 & 9 & 8 & 9 & 7 & 8 & 3 & 9 & 6 & $\mathbf{1 . 0 0}$ \\
A5 & 9 & 9 & 9 & 7 & 9 & 8 & 9 & 7 & 8 & 9 & 7 & 6 & 4 & 5 & 6 & $\mathbf{0 . 9 2}$ \\
A6 & 9 & 9 & 9 & 9 & 8 & 5 & 6 & 7 & 7 & 8 & 5 & 6 & 8 & 7 & 7 & $\mathbf{0 . 9 1}$ & \\
A4 & 9 & 9 & 9 & 9 & 9 & 8 & 9 & 9 & 8 & 9 & 2 & 6 & 3 & 7 & 3 & $\mathbf{0 . 7 9}$ & \\
A13 & 9 & 6 & 7 & 9 & 9 & 9 & 6 & 7 & 4 & 5 & 8 & 6 & 3 & 4 & 6 & $\mathbf{0 . 7 1}$ \\
A8 & 9 & 6 & 7 & 6 & 5 & 7 & 9 & 7 & 4 & 1 & 6 & 6 & 4 & 1 & 7 & $\mathbf{0 . 5 0}$ \\
A7 & 9 & 7 & 3 & 9 & 4 & 6 & 8 & 7 & 7 & 3 & 7 & 4 & 3 & 2 & 4 & $\mathbf{0 . 4 9}$ \\
A12 & 6 & 9 & 7 & 3 & 8 & 4 & 6 & 4 & 3 & 4 & 7 & 4 & 6 & 3 & 2 & $\mathbf{0 . 4 5}$ \\
A9 & 8 & 4 & 5 & 4 & 6 & 9 & 3 & 4 & 6 & 5 & 4 & 4 & 7 & 4 & 1 & $\mathbf{0 . 4 3}$ \\
A10 & 9 & 3 & 6 & 5 & 7 & 6 & 5 & 4 & 5 & 6 & 3 & 1 & 6 & 5 & 1 & $\mathbf{0 . 4 1}$ \\
A11 & 6 & 6 & 4 & 6 & 3 & 4 & 4 & 2 & 5 & 4 & 2 & 2 & 3 & 3 & 6 & $\mathbf{0 . 2 7}$ \\
A14 & 7 & 6 & 4 & 4 & 1 & 1 & 2 & 2 & 3 & 2 & 1 & 2 & 4 & 1 & 1 & $\mathbf{0 . 0 0}$ \\
\hline
\end{tabular}

standardization of education is urgently needed. Applying models of educational engineering constructed with mathematical logic in education are adequately proposed approaches. 


\subsection{Rough Set}

This paper has used ROSE2 software of Laboratory of Intelligent Decision Support Systems of the Poznan University of Technology on the URL http://www-idss.cs.put.poznan.pl (2014) [22] [23] for processing data. The data from decision table are inputted, after processing data, the results obtained including: lower approximation, upper approximation, boundary area of attributes, reducts and core of attributes. Each type of results obtained are valuable for research, depending on the purpose of research which types of result and its structural analysis will be interested.

\section{Result Analysis}

Activities in the classroom, one can see the instructor transmitting knowledge, shows the world we are living is featured in the characterization of the design, on the other hand it shows how the learners think and learn knowledge, judge and understand knowledge in thinking norms, to master the essence of things and phenomena of the world.

It is noteworthy that, the key concepts and knowledge matter to the content, characterization of the content is denoted by the world, the first thing, to acknowledge curriculum, the code is fundamental; the second thing, to identify systematic structure of knowledge manipulating the process of teaching, during shuttling back and forth such as specific vocabulary, interactivity, awareness, misunderstanding or understanding, wrong solution etc., judgment criterion manifests the truth of existence object, reality and adaptability. Based on the complexity and variability of understanding in learning, grasp and diagnostic of learning results are becoming increasingly important.

\subsection{Kernel Knowledge Structure}

Generally speaking, systematic structure of knowledge transferred in the classroom, one side based on proposition employed with expertise, the other side on academic profession, are implemented with teaching experience and interaction between teachers and students, the transformed principles as following: 1) Between concepts, there are implied relationships, difficulties, the difference in size and logic, 2) The attributes of concept for this hierarchy chain structure from concrete to abstract, from low level rise to high level, and 3) Mindset, cognitive understanding, cognitive learning process and only after understanding the concept below, to learn the advanced concepts and complex difficulties.

Based on the results outputted from ROSE2 software, the third year students and the syllabus researchers, who answered the work sheet, both of them have a simple core (Table 7), which will be dealt with, evaluated and diagnosed according to construction of grey structural modeling (see Figure 2), the full and simplified forms, provide visual structure analysis.

Table 7. Kernel structure.

\begin{tabular}{|c|c|c|}
\hline Concept $\mid$ Subject & Researcher & Student \\
\hline Epistemology & 8 & 3 \\
\hline Axiology & 6 & 3 \\
\hline Cognitive development & 6 & 7 \\
\hline Metaphysics & 6 & 2 \\
\hline Authentic and criticism & 6 & 4 \\
\hline Positive psychology & 6 & 9 \\
\hline Personality development & 4 & 8 \\
\hline Educational finance & 4 & 9 \\
\hline Structural functionalism & 4 & 6 \\
\hline System theory & 1 & 5 \\
\hline Educational politics & 2 & 8 \\
\hline Regional culture & 2 & 6 \\
\hline
\end{tabular}


Figure 3 and Figure 4 and Table 7 show that: 1) For the cognitive understanding difficulties of introduction to education, the structure of the syllabus researchers were denoted more appropriate with judgement, which is the case difficult to understand philosophy section, followed by psychology, sociology belongs to easier learning section; awareness of the third-year students is undifferentiated; 2) For the connotation and denotation of course of introduction to education, the positive psychology belongs to the mental activity of psychological class, has the individual character, philosophy, sociology then has the directions to with real life situations, each focusing on, however, psychology development cannot be divorced philosophy and sociology of norms and context; 3) The system theory, axiology and metaphysics are the basic concept structures, shaping one kind from the whole to the part, from the abstract to the concrete, ordain the thinking pattern of axiology.

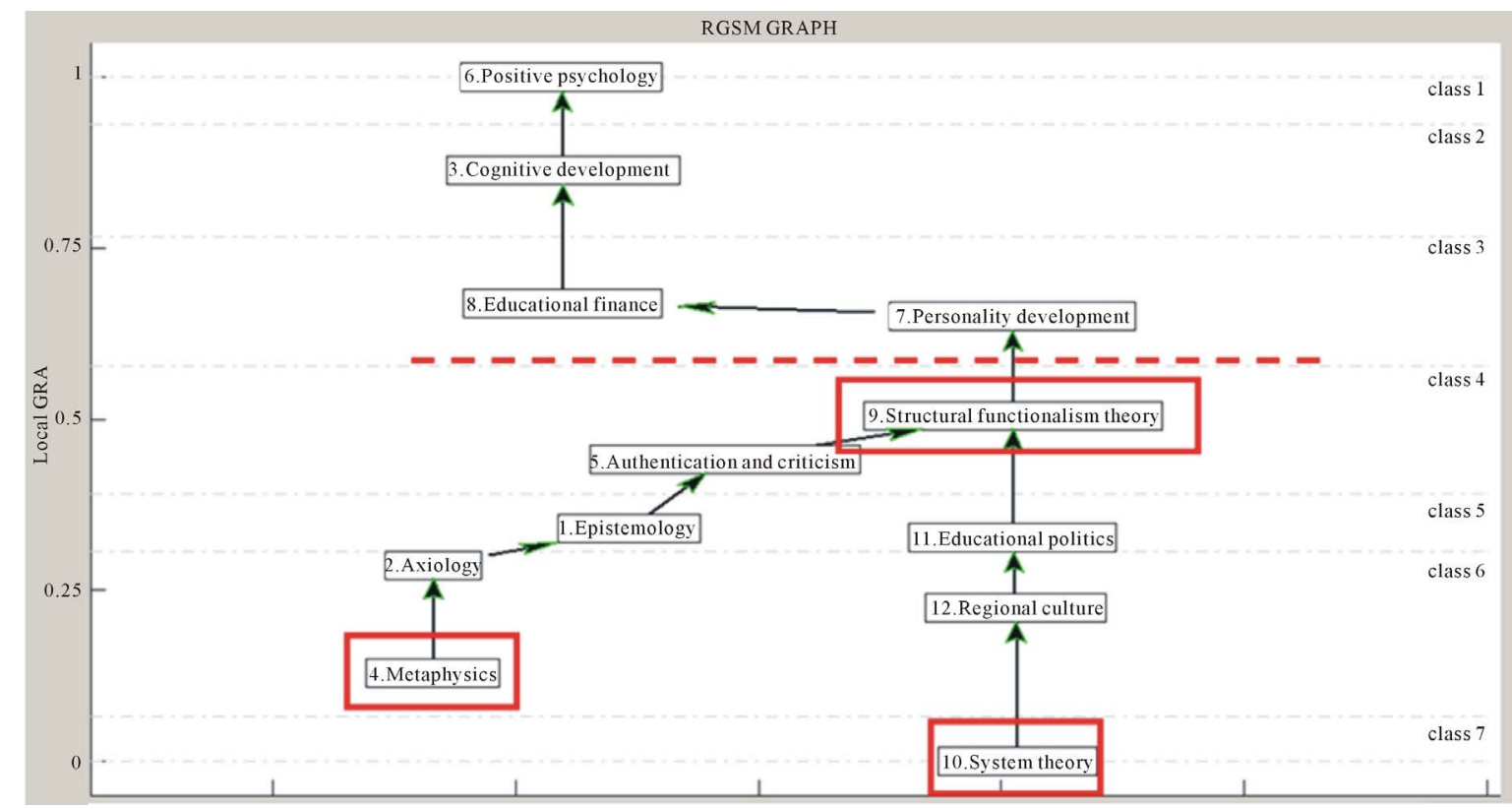

Figure 3. GSM diagram of educational student and researcher (simplified form).

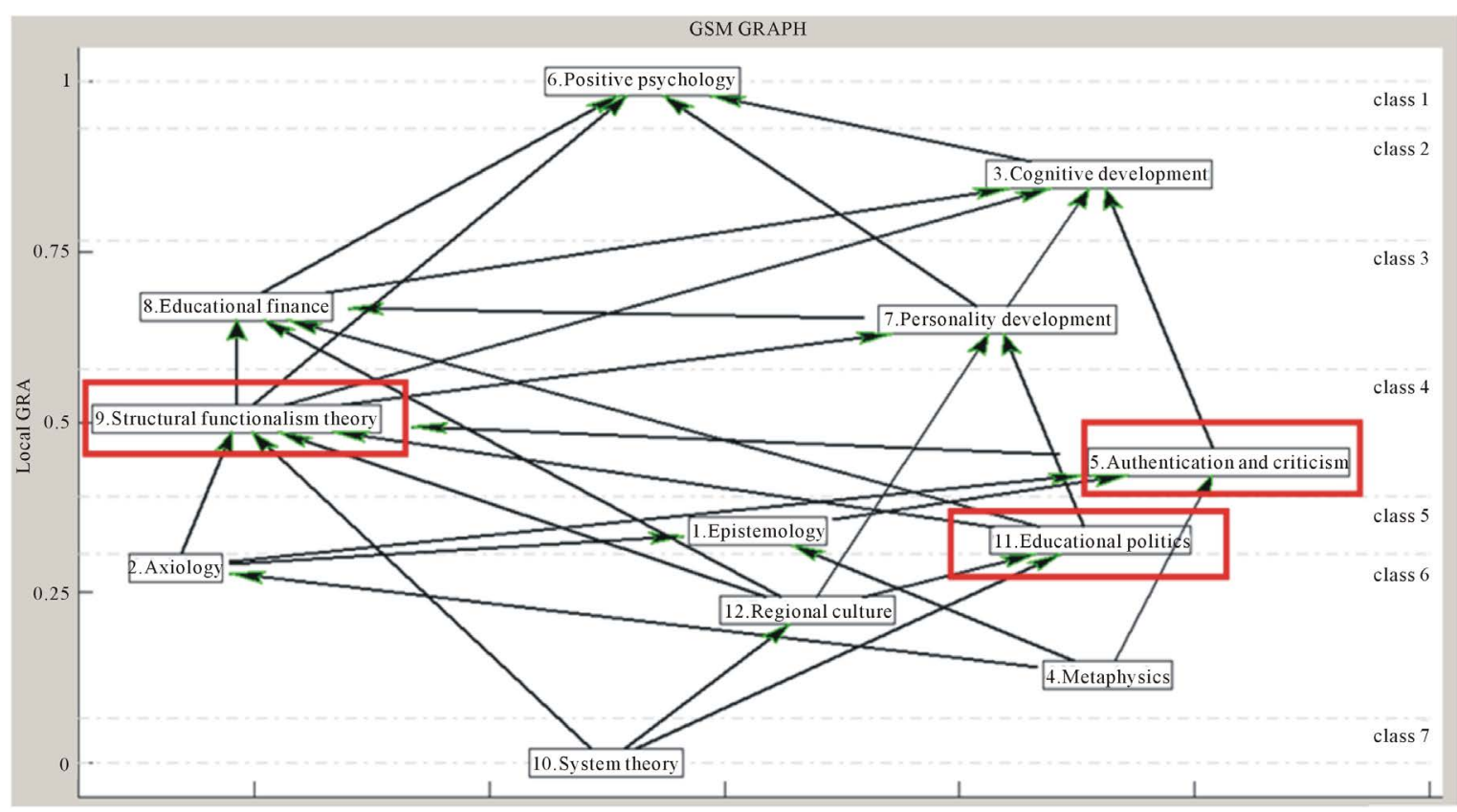

Figure 4. GSM diagram of educational student and researcher (full form). 
It is worth mentioning that, the difference between simplified form and complicated (full) form of GSM diagram are: 1) Figure 3 shows the simplified form structure which is a special case of the complicated form in Figure 4, the conceptual structure that can be captured efficiency; 2) In principle, Figure 4 can be divided into two parts, and the structural functionalism theory is an intermediary, the below of it is leaning to social science and phenomenology; the above of it is about to behavioral science; philosophy, sociology and psychology intertwined from subjective, sociability to one himself; 3) The lower part of figure includes philosophical formalism and social network aggregated to structural functionalism in sociology, both interactive as in- and exterior; the upper part is more individual-oriented psychology.

\subsection{Structural Analysis}

Firstly, Table 7 shows that: 1) There are the differences about the cognitive learning between educational undergraduates and researchers, 2) The arrangement of learning difficulties by educational researchers is converged on subjects order: philosophy, psychology, sociology, 3) Learning difficulties ordering by educational undergraduates is psychology, sociology and philosophy.

Secondly, in Figure 3 and Figure 4, the simplified and full forms of kernel GSM diagram show that: 1) The structural functionalism is convergence intersection of metaphysics and system theory, 2) The conceptual structure of locality has meaning or classification characteristics, 3) The conceptual structure of globalization is still difficult and academic knowledge systems, cognitive understanding generate correspondence relation.

Based on the sorting of number value of grey relational analysis, S9 with its Gamma $=0.48$ in the center (see Table 3) and S12 with its Gamma = 0.18 (see Table 5), This means: 1) The learning outcomes of educational undergraduates and researchers are different; 2) For the people who do not fully understand the concept planning second teaching, the gap between them are large [24]; 3) The second teaching can be implemented the small group cooperative learning, namely those who understand things more support people who understand things in middle level, the weaker ones are implemented the second teaching by the teachers with heterogeneous lesson (the text with multiple design). In order to further understand the learning situation of the second groups, the structure of grey structural modeling is used to analyze the structure.

For the third-year students:

Figure 5 and Figure 6 show that: 1) The philosophy connect with other conceptual area such as regional culture, cognitive development; 2) The learning difficulty of positive psychology is high, this intention is to promote the depth and breadth of learning, and reveal to enhance the physical, biological to mind of personal character; 3) The distinguishing attribute of knowledge and feelings, knowledge and skills, or knowledge and perception, feelings and skills have improvement space.

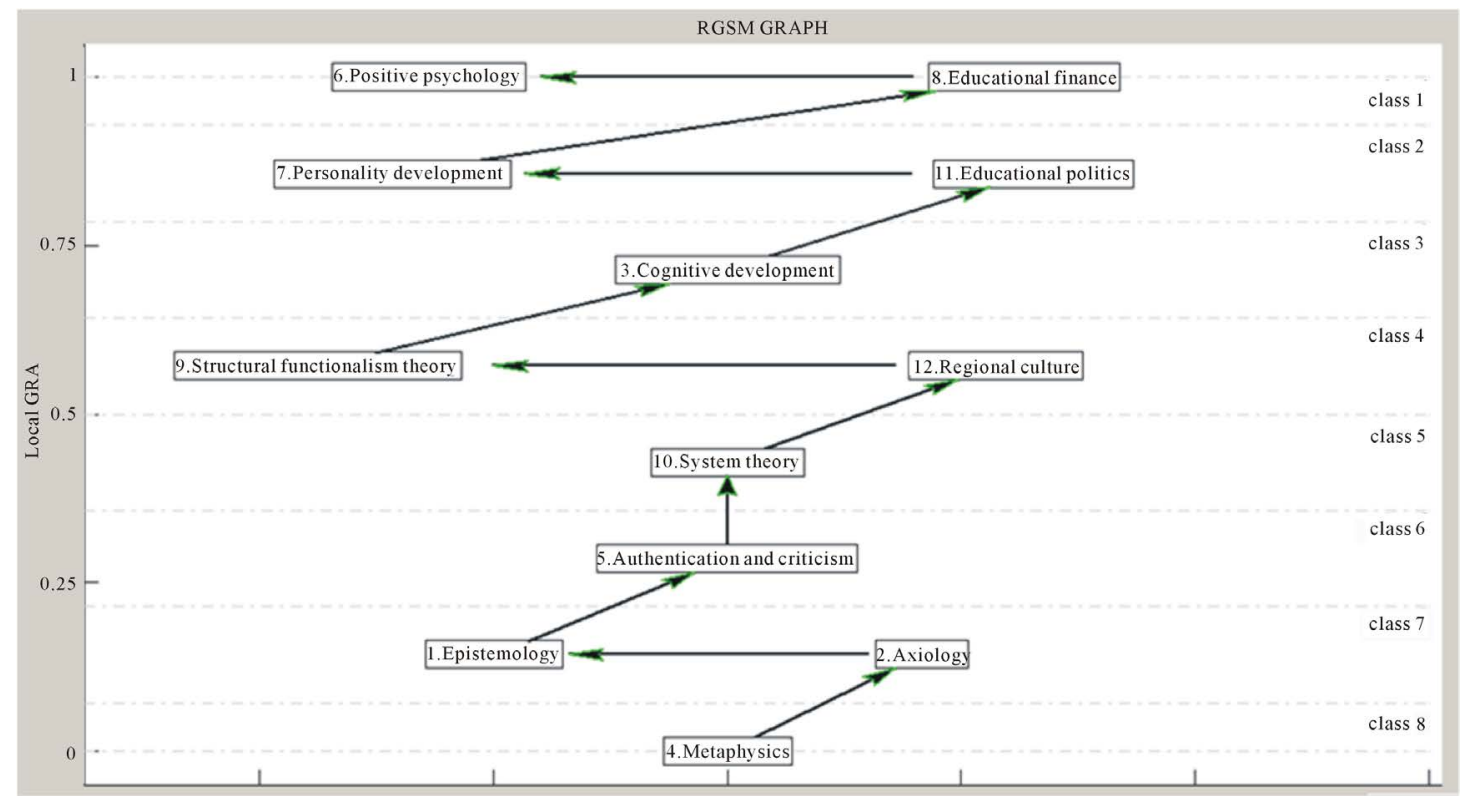

Figure 5. GSM diagram of educational student (simplified form). 


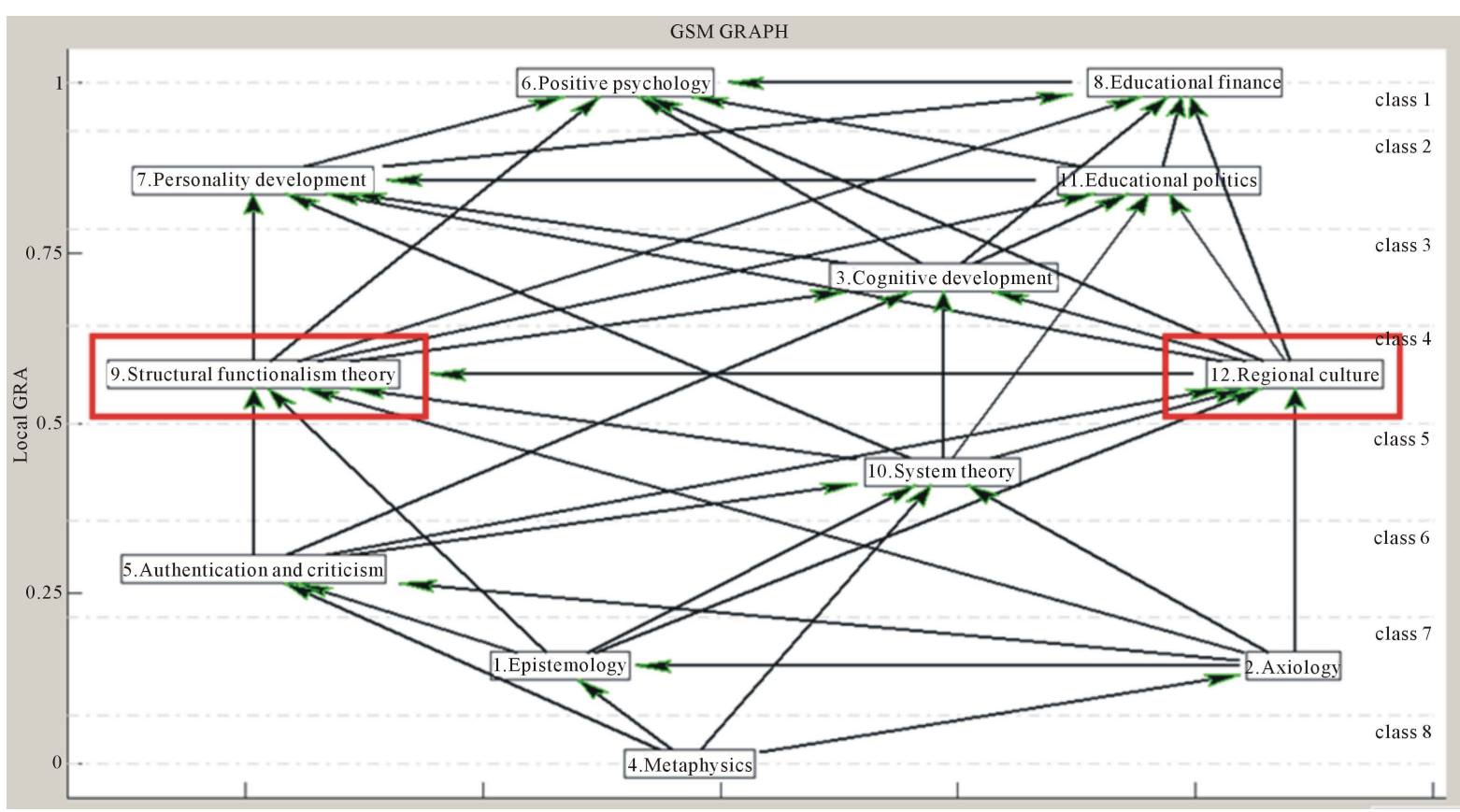

Figure 6. GSM diagram of educational student (full form).

For the syllabus researchers (master degree program):

Figure 7 and Figure 8 show that: 1) The conceptual compatibleness between sociology and psychology must have logical relationship; 2) Philosophy isolated from the system, only the authentication and criticism element, epistemology, and axiology are associated; and 3) Based on the philosophy is the mother of learning, the relationship between philosophy and sociology is very close, "I am existing, therefore I think," and vice versa, "I am thinking, therefore I exist,” reinforcing path such as educational politics supporting on axiology, structural functionalism theory supporting on epistemology, metaphysics must have close relationship with psychology.

Synthesis of descriptions above (Figures 5-8), obtained: 1) Educational students and researchers have cognitive gap between them; 2) The special cases in simplified and full forms are possible unified; 3) The vertical direction in full and simplified forms can master the focal points of overall structure, or represent the starting point of learning advance, or making as second teaching, remedial teaching basis, or even on the table in mind the issue of distribution at below or above key points.

\subsection{Structural Characterization}

Combining Nagai's matrix based structure modeling and graph theory, in order to construct the structural analysis of integrity and visualization (see Figure 9), in the first place, it is helpful for communication and diagnostic, in the second place, according to the strategic plan the action is performed. Next, to compare and grasp the knowledge structure development of introduction to education between educational undergraduates and researchers based on the differences of number value, one place Figure 5, Figure 6 and Figure 9 are the reference basis, the other place, the academic development structure in correspond to the professional consensus and effective academic norms is aimed at.

Finally, the point concepts of convergence and differentiation are: 1) The place where the conversion and transformation of knowledge hierarchy structure exist, 2) Teaching and learning are possible difficulties, 3) Making every effort for the same class with heterogeneous structure.

\section{Conclusions}

"For great education plan, teacher must be centered". Introduction to education is not only one of the educational compulsory subjects, but also is a subject of structural and scientific cognitive education. With the broad scope and the main academic knowledge system of higher education, teaching and learning quality have become 


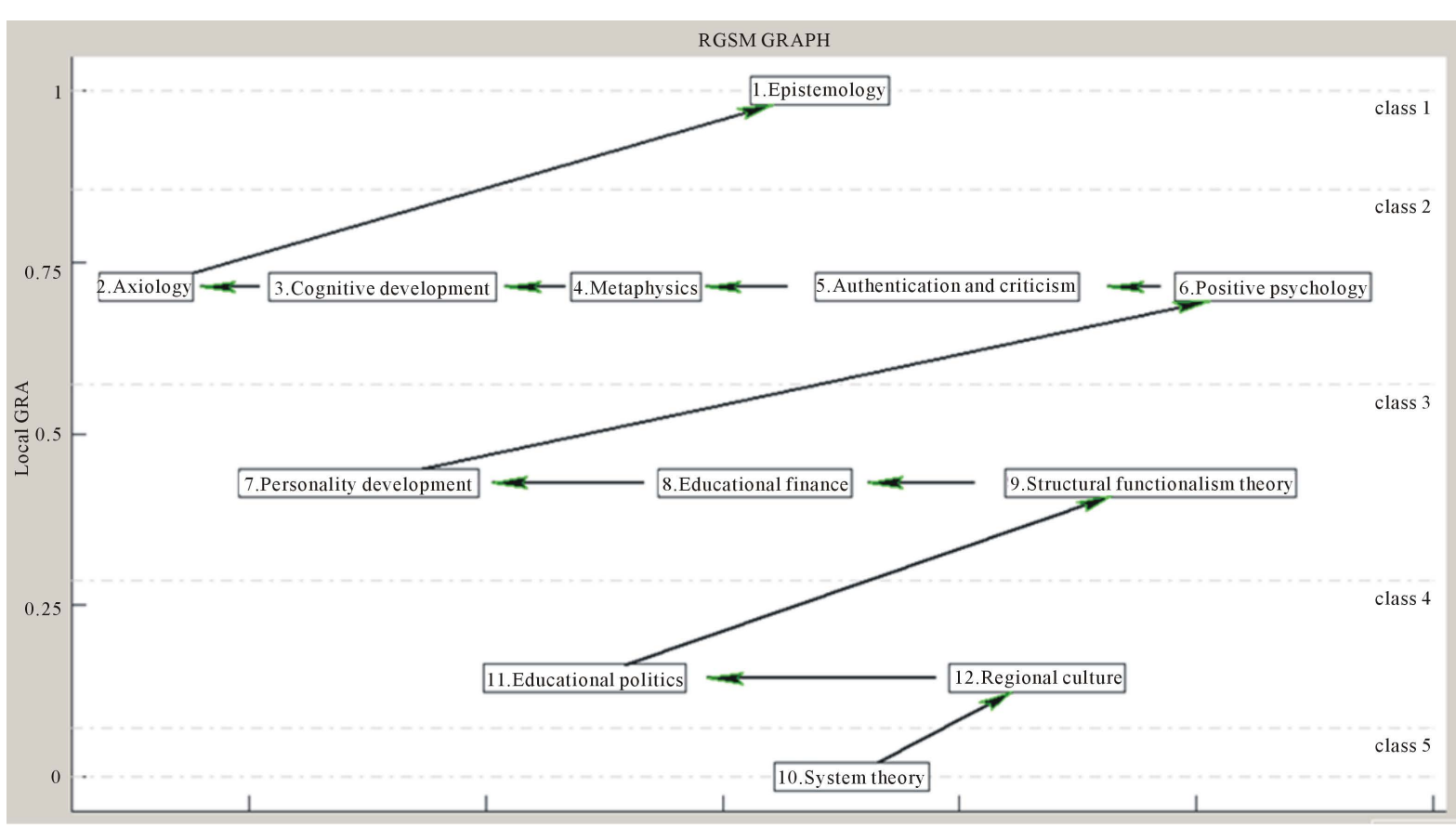

Figure 7. GSM diagram of educational researcher (simplified form).

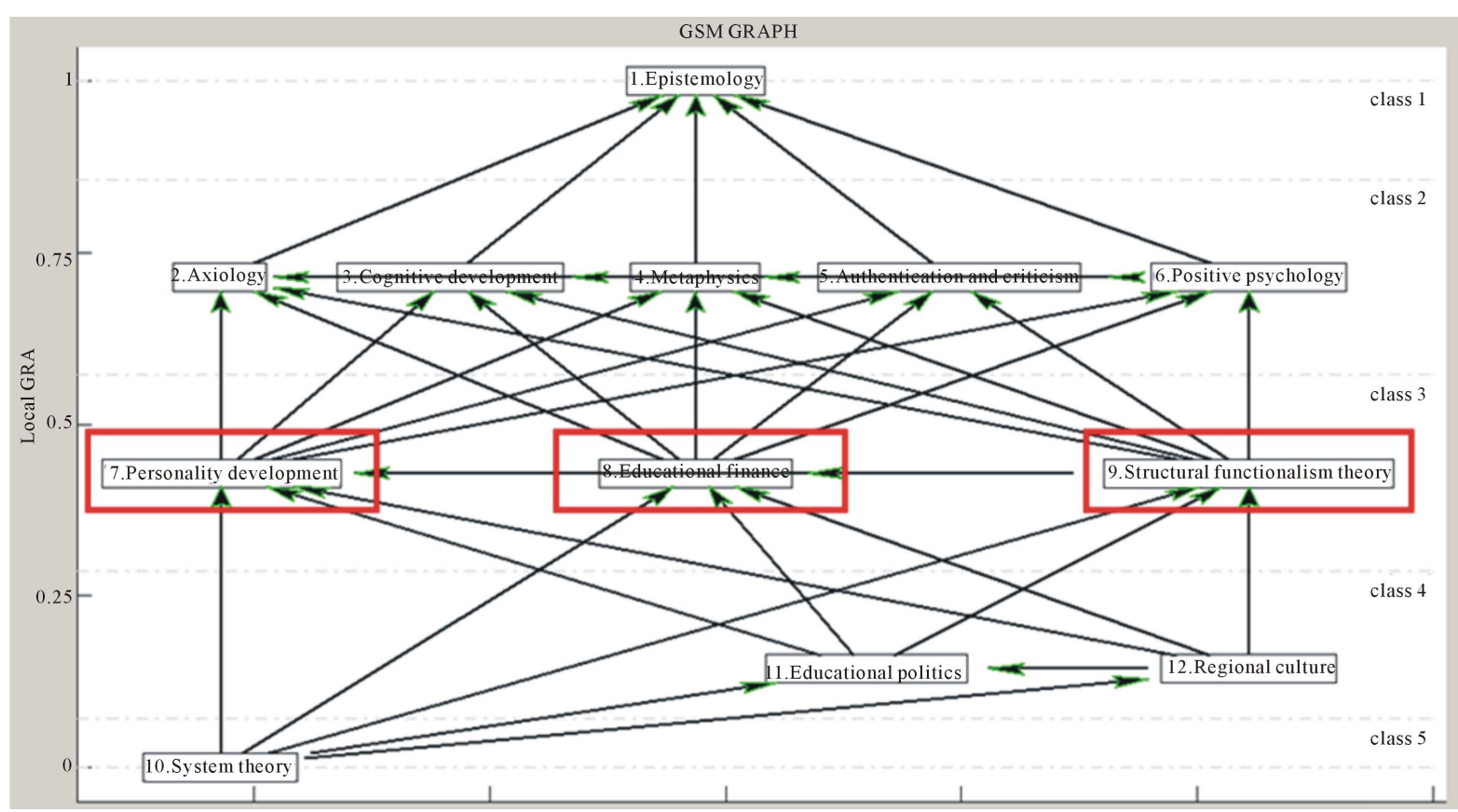

Figure 8. GSM diagram of educational researcher (full form).

the focus, so it takes the learning outcomes of the third year undergraduate educational students and master degree program researchers in primary education course to analyze for understanding. Aggregated by the concepts, the questionnaire respondents have been processed by Nagai's grey relational analysis program, ROSE 2 software, and technique as indicated in Tables 3-6 and Figures 2-9.

Based on the systematic instructional design principles, the starting behavior of learners are based on the same class with heterogeneous structure teaching on classroom, effective control, diagnose this starting behavior is imperative. The conclusion from Figures 5-8: 1) The thinking pattern is the inductive and deductive cycles, 2) 


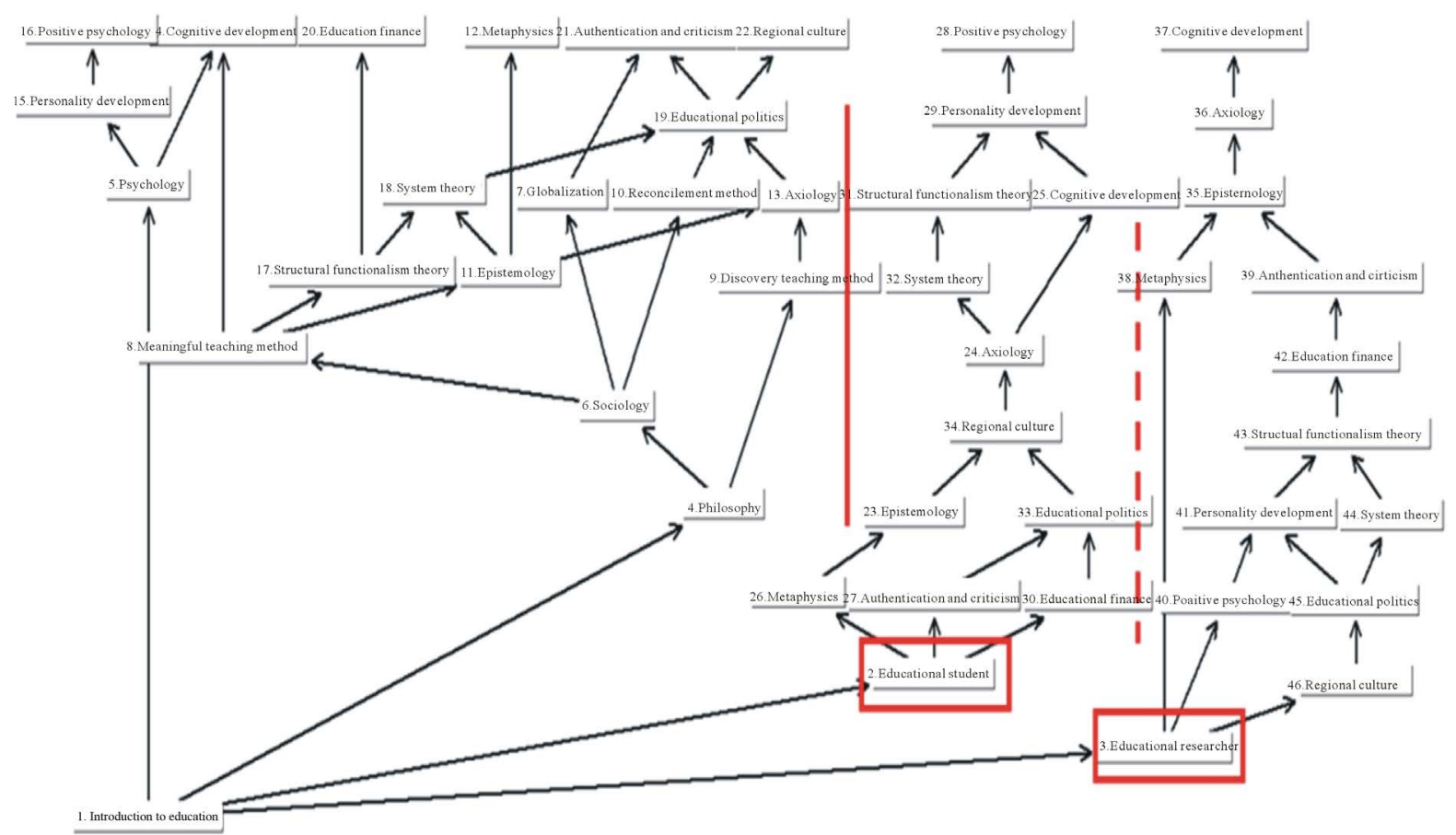

Figure 9. MSM diagram of introduction to education.

The detail concepts converge in inductive point, from a point it can be deduced for expanding differentiation, and spiraling to construct knowledge system; 3) The focal point of directed hierarchical structure can be considered the diagnosis, grouping, evaluation, and making every effort of second education.

Finally, since the 1970s, academic education and professional development have become the norm, enhancing the educational research methodology and research method has become necessary. The combination mathematical tools with professional consensus on education in research are that the data collection and calculation, interpretation of knowledge structure and logical implication, along with systemization and digitalizing to achieve the purpose of prediction and control. Now, the use of mathematical tools to construct the context of situation of confidence interval; using Rough set to reveal the concepts in core, using LGRA to select adjacent learners and construct GSM, MSM combining with graph theory to characterize and visualize the multiple sets structure, it can be seen the following benefits: 1) Scientifically and diagnostically demo learning results between the two groups, 2) Divergence and gap emerges in structural analysis, 3) Structural analysis displays in quantization and visualization.

\section{References}

[1] Ho, H.C. (2012) Reconstructing Taiwan's Primary Teacher Education in Academic University—Primary Teacher Education Institutionalized. Hong Kong Teachers' Centre Journal, 11, 197-216.

[2] Ho, H.C., Wang, X.Z., Chung, C.Y., Sheu, T.W. and Nagai, M. (2013) Applying ATLAS.ti, 5W1H and ISM to Study the Proposal of Constructing Elementary School Teacher's Specialties with the Text with Multiple Design and the Perspective of Teaching Styles. Journal of Teacher Education, 2, 53-72.

[3] Ho, H.C., Pham, D.H., Shih, P.C., Sheu, T.W. and Nagai, M. (2014) Based GSP Chart, Rasch Model GSP Curve and GSM to Analyze Math Test-An Example on $6^{\text {th }}$ Graders' Math Subject Test. Journal of Educational Measurement and Statistics, 22, 25-65.

[4] Ho, H.C. and Nagai, M. (2015) Academic Capitalism, Knowledge Function Only. Taiwan Education Review Magazine, 4, 51-60.

[5] Pawlak, Z. (1982) Rough Sets. International Journal of Information and Computer Science, 11, 341-356. http://dx.doi.org/10.1007/BF01001956

[6] Deng, J. (1989) Introduction to Grey System. The Journal of Grey System, 1, 1-24.

[7] Wen, K.L., Nagai, M., Chang, T.C. and Wang, H.C. (2008) An Introduction to Rough Set Theory and Application. 
Chuan Hwa Publishing Ltd., Taipei.

[8] Pawlak, Z. (1991) Rough Sets: Theoretical Aspects of Reasoning about Data. Vol. 9, Springer. http://dx.doi.org/10.1007/978-94-011-3534-4

[9] Walczak, B. and Massart, D. (1999) Rough Sets Theory. Chemometrics and Intelligent Laboratory Systems, 47, 1-16. http://dx.doi.org/10.1016/S0169-7439(98)00200-7

[10] Nagai, M., Yamaguchi, D. and Li, G.D. (2005) Grey Structural Modeling. Journal of Grey System, 8, 119-130.

[11] Yamaguchi, D., Li, G.D., Akabane, K.M.T. and Kitaoka, M. (2007) A Realization Algorithm of Grey Structural Modeling. Journal of Grey System, 10, 33-40.

[12] Yamaguchi, D., Li, G.D. and Nagai, M. (2007) Verification of Effectiveness for Grey Relational Analysis Models. Journal of Grey System, 10, 169-181.

[13] Nagai, M., Tsai, C.P. and Chen, T.L. (2013) Matrix Based Interpretative Structural Modeling. Proceedings of the 5th International Conference on Technology and Mathematics Education and Workshop of Mathematics Teaching, 509-518. (In Chinese)

[14] Nagai, M. and Tsai, C.P. (2013) Matrix Based Interpretative Structural Modeling. International Journal of Kansei Information, 4, 159-174.

[15] Ho, H.C., Chiang, H.J., Liao, S.L., Kung, K.D. and Nagai, M. (2013) A Study of Assessment on Educational Philosophy with FCA and MSM-A Case Study on Selected Units of Philosophy. Proceedings of 2013 International Conference on Grey System Theory and Kansei Engineering Conference, 212-221. (In Chinese)

[16] Ho, H.C., Sheu, T.W. and Nagai, M. (2013) A Study of Constructing an Epistemological Entity and Cognition Patterns with FCA, Rough Sets and MSM-A Case Study on an Educational Philosophical Assessment. Proceedings of the Chinese Association of Psychological and Educational Testing 2013 Annual Conference. (In Chinese)

[17] Friedman, T.L. (2006) The World Is Flat: The Globalized World in the 21st Century. Updated and Expanded Edition, Penguin Group, New York.

[18] Warfield, J.N. (1974) Toward Interpretation of Complex Structural Modeling. IEEE Transportation Systems Man Cybernet, 4, 405-417. http://dx.doi.org/10.1109/TSMC.1974.4309336

[19] Bryman, A. and Cramer, D. (1997) Quantitative Data Analysis with SPSS for Windows: A Guide for Social Scientists. Routledge, London.

[20] Nagai, M. and Yamaguchi, D. (2004) Grey Theory and Engineering Application Method. Kyoritsu Publisher, Tokyo. (In Japanese)

[21] Western Association of Schools and Colleges (2015) ACS WASC Overview. http://www.acswasc.org/wasc/acs-wasc-overview/

[22] Predki, B., Słowiński, R., Stefanowski, J., Susmaga, R. and Wilk, S. (1998) ROSE-Software Implementation of the Rough Set Theory. In: Polkowski, L. and Skowron, A., Eds., Rough Sets and Current Trends in Computing, Springer, Berlin, 605-608.

[23] Prędki, B. and Wilk, S. (1999) Rough Set Based Data Exploration Using ROSE System. In: Ras, Z.W. and Skowron, A., Eds., Foundations of Intelligent Systems, Springer, Berlin, 172-180. http://dx.doi.org/10.1007/bfb0095102

[24] Ho, H.C. and Nagai, M. (2015) Active Teaching, Lively Teaching? Cooperative Learning? Education Standardization! Taiwan Education Review Magazine, 4, 59-70. (In Chinese) 